\title{
CRYOTE (Cryogenic Orbital Testbed) Concept
}

\author{
Mari Gravlee ${ }^{*}$ and Bernard Kutter ${ }^{\dagger}$ \\ United Launch Alliance, Centennial, $\mathrm{CO}$ \\ Mark Wollen \\ Innovative Engineering Solutions, Murrieta, CA \\ Noah Rhys ${ }^{\S}$ \\ Yetispace, Inc., Huntsville, AL \\ Laurie Walls* \\ NASA Kennedy Space Center, FL
}

Demonstrating cryo-fluid management (CFM) technologies in space is critical for advances in long duration space missions. Current space-based cryogenic propulsion is viable for hours, not the weeks to years needed by space exploration and space science. CRYogenic Orbital TEstbed (CRYOTE) provides an affordable low-risk environment to demonstrate a broad array of critical CFM technologies that cannot be tested in Earth's gravity. These technologies include system chilldown, transfer, handling, health management, mixing, pressure control, active cooling, and long-term storage.

United Launch Alliance is partnering with Innovative Engineering Solutions, the National Aeronautics and Space Administration, and others to develop CRYOTE to fly as an auxiliary payload between the primary payload and the Centaur upper stage on an Atlas $V$ rocket. Because satellites are expensive, the space industry is largely risk averse to incorporating unproven systems or conducting experiments using flight hardware that is supporting a primary mission. To minimize launch risk, the CRYOTE system will only activate after the primary payload is separated from the rocket. Flying the testbed as an auxiliary payload utilizes Evolved Expendable Launch Vehicle performance excess to cost-effectively demonstrate enhanced CFM.

\begin{tabular}{llll} 
& \multicolumn{3}{c}{ Nomenclature } \\
ACES & Advanced Common Evolved Stage & IES & Innovative Engineering Solutions \\
ACS & Attitude Control System & LAD & Liquid Acquisition Device \\
Centaur & Upper stage of Atlas V EELV & LH2 & Liquid Hydrogen \\
CFM & Cryogenic Fluid Management & MLI & Multi-layer Insulation \\
EDS & Earth Departure Stage & Rideshare & Launching as an auxiliary payload \\
EELV & Evolved Expendable Launch Vehicle & TRL & Technology Readiness Level \\
ESPA & EELV Secondary Payload Adapter & TVS & Thermodynamic Vent System \\
g & Gravity & ULA & United Launch Alliance
\end{tabular}

\footnotetext{
* Sr. Mechanical Engineer, Advanced Programs, United Launch Alliance, Denver, CO 80127, AIAA Senior Member

${ }^{\dagger}$ Sr. Staff, Manager Advanced Programs, United Launch Alliance, Denver, CO 80127, AIAA Senior Member

${ }^{\ddagger}$ Vice President, Innovative Engineering Solutions, Murrieta, CA 92562, AIAA Member

${ }^{\S}$ Senior Engineer, Yetispace, Inc., Huntsville, AL 35802, AIAA Member

** NASA Launch Services Program, Thermal/Fluids Group VA-H3, Kennedy Space Center, FL 32899, AIAA Senior Member
} 


\section{Introduction}

The space industry is primed for the next big leap - for humans to go farther than we have ever gone before in space. Arguably the most efficient form of propulsion in space for long distances over relatively short time periods is chemical propulsion using cryogenic propellants. The high Isp and thrust available from cryogenic propellants can produce the required amount of energy to travel to the moon in four days and Mars in less than nine months. However, the undeveloped state of the technologies needed to handle cryogenic fluids during long low-gravity maneuvering offsets the performance advantages. Current space based cryogenic propulsion is viable for hours, not the weeks to years needed by NASA Space Exploration and the space science community. Ongoing efforts to advance in-space cryo-fluid management (CFM) capabilities have languished for decades, partially due to lack of affordable space access for flight demonstration. The lack of flight-demonstrated CFM has forced NASA's Exploration initiative to resort to alternative solutions (storables and short durations), severely impacting mission capabilities and likelihood of mission success.

United Launch Alliance (ULA), in partnership with NASA and industry, is developing an affordable CRYogenic Orbital TEstbed (CRYOTE) to demonstrate a broad array of critical CFM technologies in space. These technologies include: system chilldown, transfer, handling, health management, mixing, pressure control, active cooling and long-term storage. Testing and validation in the micro-gravity environment is essential for developing improved mission capabilities using cryogenics. Results from CRYOTE experiments will aid in the development of enhanced upper stages, Earth Departure Stage (EDS), lunar lander, cryogenic propulsion modules, propellant depots, solar thermal and nuclear thermal propulsion systems, small satellite hydrogen propulsion, and cryogenic science applications.

CRYOTE's affordability is achieved by using Evolved Expendable Launch Vehicle (EELV) Rideshare opportunities to avoid dedicated launch costs. Residual liquid hydrogen (LH2) from the rocket's upper stage is used to minimize risk to a primary mission. In early 2009, ULA visited several NASA field centers to gauge support for the advancement of CFM technologies. The response was overwhelming. Three NASA field centers have become active participants in the development of the CRYOTE system, and several others have expressed interest in utilizing the testbed for NASA experiments. In addition, research and development funding from across industry has supported the development of the CRYOTE concept. Full funding for CRYOTE is now required for commitment to Rideshare opportunities.

\section{CRYOTE Design Overview}

CRYOTE is a long-duration in-space laboratory containing a sizeable tank of LH2. The laboratory is installed on an Atlas $\mathrm{V}$ rocket

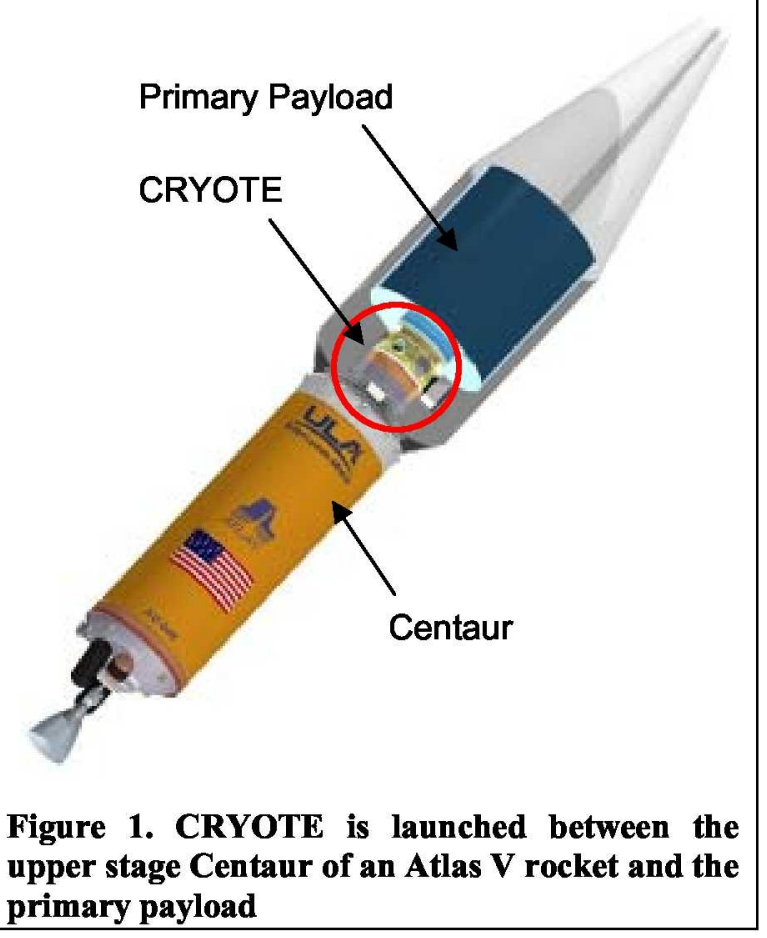
between the upper stage Centaur and the primary payload (Figure 1). To minimize risk to the primary payload, the CRYOTE tank is launched dry and with dormant avionics. Following delivery of the primary payload, CRYOTE's avionics are activated by Centaur's flight computer. The pyro valve separating Centaur's propellant tank from the CRYOTE fluid line is fired, and a settling sequence chills the system and delivers residual LH2 from the Centaur upper stage to fill the CRYOTE tank. After CRYOTE is filled with Centaur's residual LH2, the two crafts separate, leaving CRYOTE as an independent free flyer. 
There are several drivers that contribute to CRYOTE's design. Structural stability, vibrational modes, thermodynamics, fluid motion under various gravitational environments, interface with the Centaur LH2 tank, and fluid transfer and experiment operations must all be considered. Components integral to the CRYOTE system are an EELV Secondary Payload Adapter (ESPA) ring, a tank, a skirt (which attaches the tank to the ESPA ring), a fluid regulating system, fluid lines, a robust insulation system, and an avionics suite that includes an attitude control system (Figure 2).

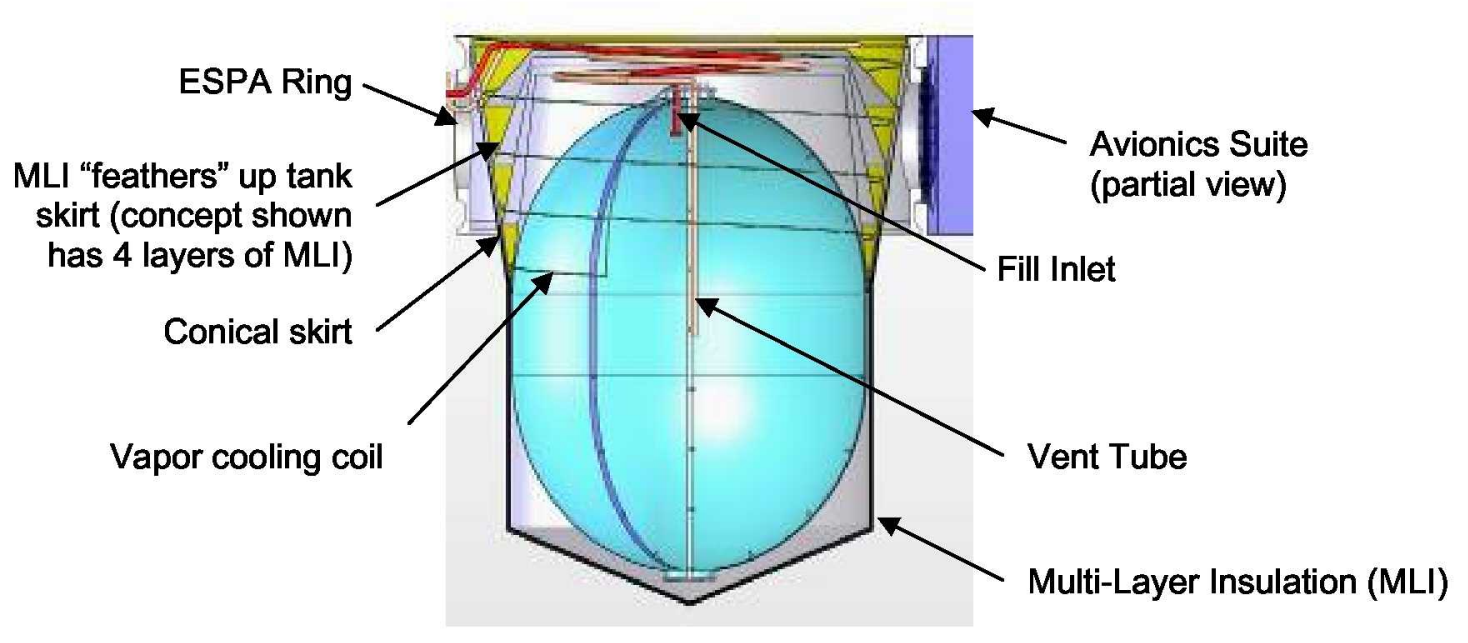

Figure 2. CRYOTE Main Components (Credit: IES)

\section{A. ESPA Ring, Tank, and Skirt}

The backbone structure of CRYOTE is an ESPA ring that is flight-qualified to carry auxiliary payloads on EELV missions. The use of an ESPA ring as the backbone to the CRYOTE structure provides flexibility. Two of the ESPA ring's six ports are utilized for basic CRYOTE functions - one port for the fluid regulating system and one port for an avionics suite. The four open ESPA ports can hold a variety of cryogenic experiments and other auxiliary payloads.

The ESPA ring is flight-proven, having carried auxiliary payloads on two missions - STP-1 in 2007 and LCROSS in 2009. The CRYOTE design mimics the LCROSS design, where the ESPA sits atop a separation system which is attached to the $\mathrm{C}$-adapter, the standard cylinder that interfaces the upper stage to the primary payload (Figure 3).

The CRYOTE tank, hung in the center of the ESPA ring, is equipped to hold a few hundred pounds of LH2. The tank material is a parameter that can be varied to support future applications. The tank will have a flange on one end large enough to install instrumentation inside the tank. Tank diameter is limited by the 60 " inner diameter of the ESPA ring in addition to the space needed for its attachment skirt and the multi-layer insulation (MLI) blanket between the tank and ESPA. A candidate flight tank donated by NASA JPL is shown in Figure 4. One end of the tank has an 8 " opening large enough to allow installation of instrumentation inside the tank.

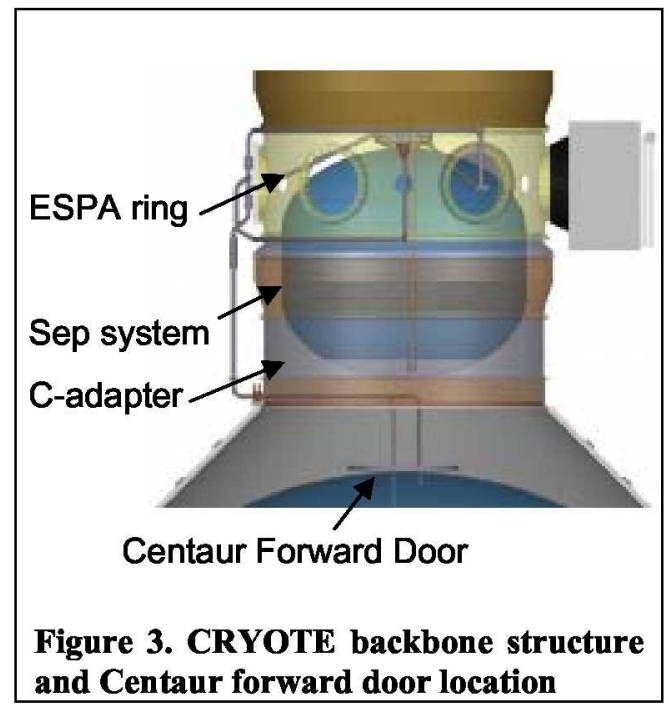


Because of the similarity to existing upper stages, Advanced Common Evolved Stage (ACES) ${ }^{1}$, and EDS, a skirt design was selected to attach the CRYOTE tank to the ESPA ring. Because the tank is launched dry, the skirt structural requirement under launch loads is driven by only the empty weight of the tank. Several skirt designs (Figure 5) were explored before the conical skirt design in Figure 2 was chosen. Preliminary thermal analysis shows that a composite skirt will have acceptable thermal efficiency and appropriate structural characteristics. To further reduce penetration heat leak, a vapor cooling coil is wrapped around the skirt.

\section{B. Insulation System}

The insulation system is key to CRYOTE's extended duration functionality. The system consists of MLI, a low-thermal-conductivity vapor-cooled tank support skirt, long "heat conduction" paths for any tank penetrations, and a Thermodynamic Vent System (TVS). The MLI system completely covers the tank and "feathers" up the tank skirt and all penetrations to eliminate thermal shorts in the MLI (Figure 2). The discharge from the TVS system spirals up the skirt to intercept heat transfer through the skirt and carry it overboard. Basic thermal analysis has been conducted to determine the type and quantity of MLI. Solutions for MLI venting during launch are being considered. Heat leak can be further reduced by using a sunshield (Figure 6). CRYOTE insulation is intended to mimic large orbital propulsion stages, providing designers with improved analysis tools.

\section{Fluid Regulating System}

The fluid regulating system, which includes cryogenic valves populating one ESPA port, controls the flow of fluid from the Centaur LH2 tank to the CRYOTE tank, the reverse settling vent system, and the TVS. The fluid line interface to Centaur is through the LH2 tank forward door (Figure 7). The fluid line schematic further details the system (Figure 8). Redundant valves on the fluid line between Centaur's LH2 tank and CRYOTE ensure mission safety while the primary payload is still attached.

\section{Avionics Suite}

The avionics suite, which populates another ESPA port, includes a flight computer, a long-duration power source, two-way communications, and an attitude control system (ACS). Integrated into a single module,

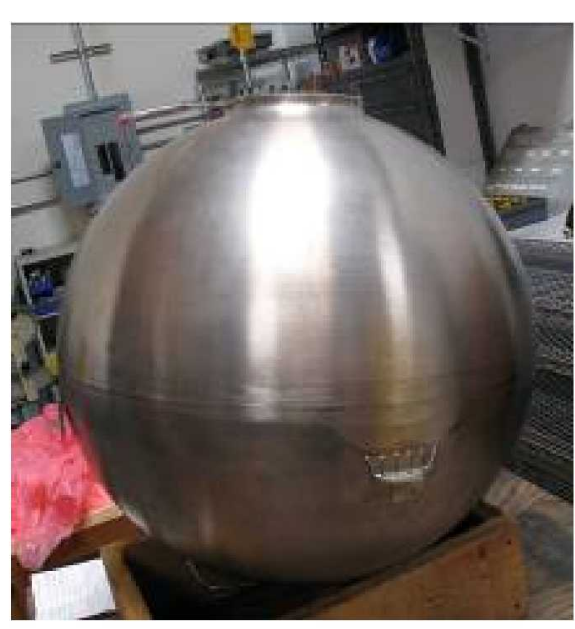

Figure 4. Potential flight tank donated to the CRYOTE project by NASA JPL (Credit: IES)

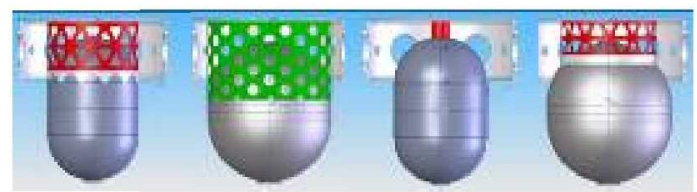

Figure 5. Previous skirt designs explored before conical skirt design was chosen (Credit: IES)

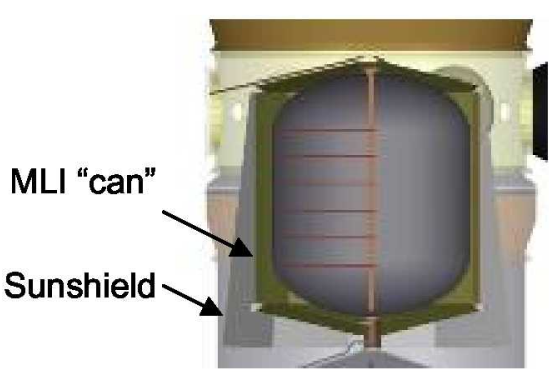

Figure 6. Alternate insulation concept with added sunshield

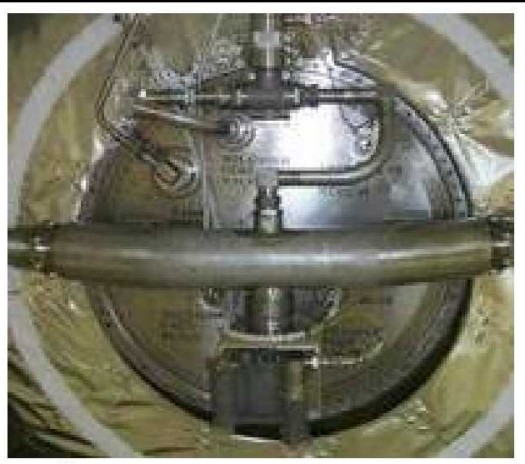

Figure 7. Centaur Fwd Door

the avionics system contains the computer for controlling the CRYOTE experiments with closed loop feedback from various instruments and for compressing and downloading the data. Due to its long flight 
duration and the likelihood of multiple independent tests, it can be reprogrammed to suit the needs of the investigators. The system also provides required housekeeping duties such as maintaining vehicle attitude, managing power, and maintaining communication links. To mitigate risk to the primary mission, CRYOTE's avionics system is disabled until Centaur's flight computer enables it after the primary payload is separated.

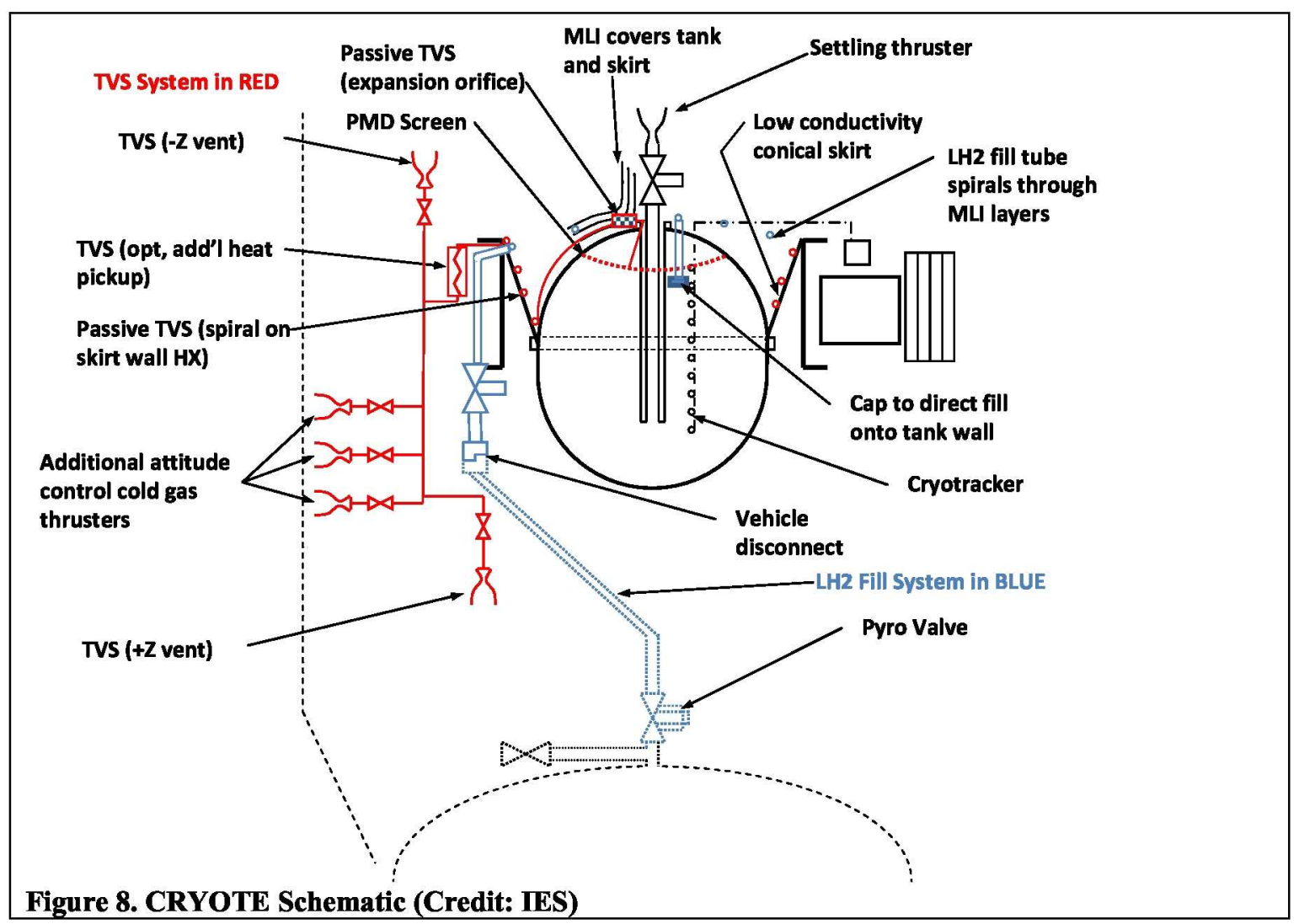

\section{E. Fluid analysis}

Preliminary tank chilldown and transfer modeling has been performed to determine chilldown sequence and line diameter requirements for the fill and vent lines. Following delivery of the primary payload, cold hydrogen gas from Centaur's LH2 tank is vented forward through the CRYOTE tank to pre-chill the CRYOTE system. This forward gas venting pushes Centaur backwards and is referred to as reverse settling. Preliminary analysis shows that the reverse settling is adequate to push Centaur's residual LH2 to the top of Centaur's LH2 tank (Figure 9). LH2 is then pulsevented through CRYOTE's tank in preparation for LH2 transfer. Once CRYOTE is chilled, residual Centaur LH2 is transferred to CRYOTE while stable pressure levels in both Centaur and CRYOTE are maintained.

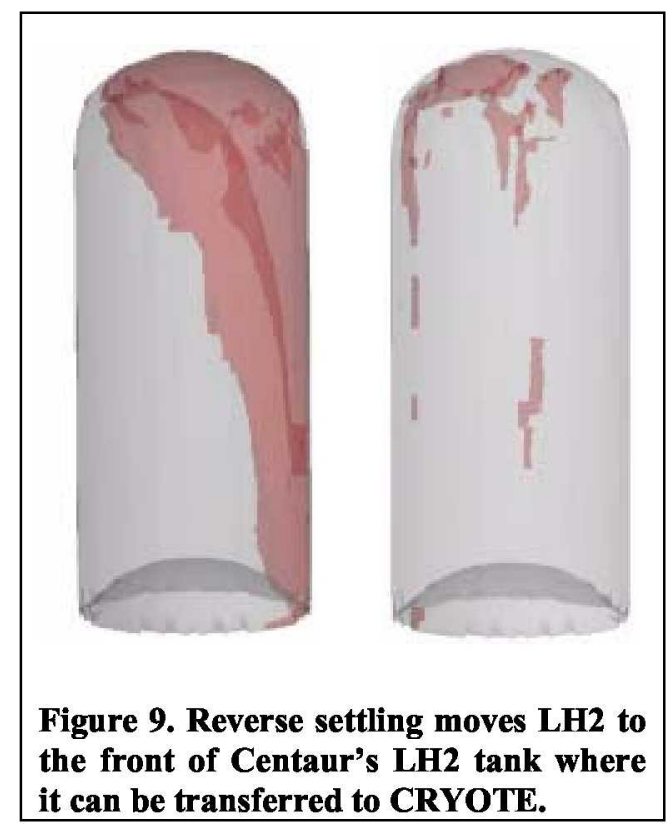




\section{F. Thermal Analysis}

Figure 10 shows a thermal analysis model that has been used to develop a basic understanding of the driving heat transfer features of the CRYOTE system. More detailed analyses will be performed once additional design details are finalized. Preliminary analysis indicates that relatively straightforward composite skirt design, on the order of 20 layers of MLI, and reasonable attention to fluid and instrumentation penetrations will allow weeks of operation before the majority of a full $\mathrm{LH} 2$ tank is lost to boiloff.

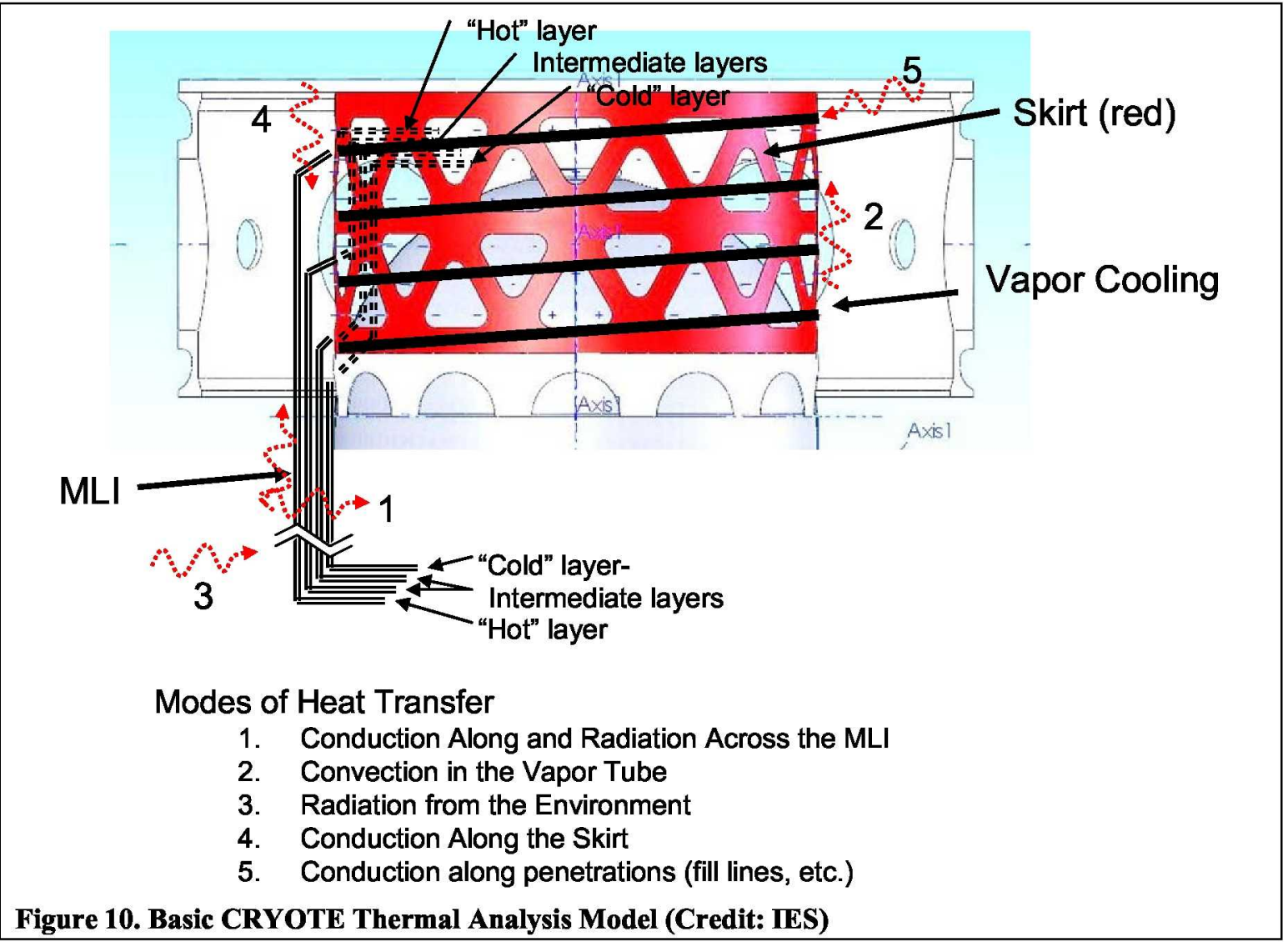

\section{G. Testing}

Testing of a ground prototype is anticipated for early 2010 in a vacuum chamber with liquid nitrogen at NASA Marshall Space Flight Center. Initial testing will be focused on fluid/mechanical systems, tanking, and insulation. The transfer operation will be demonstrated to the extent possible in a 1-g environment, and the insulation system performance will be evaluated. Thermal and fluid modeling can also be validated with the test results.

\section{CRYOTE Benefits}

\section{A. Current Cryogenic Fluid Management (CFM) State-of-the-Art}

The history of flying cryogenic stages guides the extension of existing stages to longer durations and propulsion enhancements. Some in-flight experiments can be undertaken on near-term flights, such as the centrifugal settling demonstration on the upcoming Atlas V Defense Meteorological Satellite Program (DMSP) mission (Initial Launch Capability August 2009). However, many holes exist in the CFM 
understanding and modeling capability needed to support transformational cryogenic stage development. In the mid-1960's, flight qualification for Centaur revealed CFM issues that were not evident from fluid modeling:

It was determined that the launch window for the Surveyor missions could be significantly enhanced by adding an orbital coast and an engine restart in low gravity. Based on the available information a test flight designated AC-4 was conducted. Two $8.90 \mathrm{~N}$ thrusters (producing a Bond number of 240 in the hydrogen tank) were added to the basic Centaur to provide settling thrust throughout coast. However, propellant disturbances at the main engine cut-off caused liquid entrainment in the vent. As a result the spacecraft tumbled out of control. To correct these difficulties a number of new systems were added to a second test flight designated as AC-8. The deciding parameter turned out to be the slosh transient at the start of the low gravity coast. To provide a quick fix, all possible sources of the problem were addressed and then tested together. Unfortunately, a systematic investigation of each problem to determine the real culprits and the minimum requirements for correcting the problem were not undertaken. Due to the cost and complexity of flight testing, once AC-8 proved that the problems were addressed, no further investigation of the boost phase surge was conducted. ${ }^{2}$

During the subsequent four decades and over 180 Centaur flights, our CFM capabilities steadily grew through flight experimentation and mission demands. The Titan Centaur D-1TR flights of the 1970's provided enhanced understanding by flying a Christmas tree-shaped thermal instrument suite inside of the Centaur LH2 tank to measure stratification and ullage mixing. This flight also performed five additional demonstration RL-10 burns following the two mission burns to demonstrate extensibility of the RL-10 ${ }^{3}$. Shuttle Centaur development took CFM to a whole new level with the demanding requirements to support 1 week on orbit in the Shuttle cargo bay prior to supporting on-orbit mission maneuvers. Shuttle Centaur resulted in the development of thick MLI, a thermodynamic vent system (TVS), and a pulse pump mixer to homogenize the cryogenic fluid. Sadly, the aftermath of the Challenger disaster resulted in cancellation of the Shuttle Centaur program shortly prior to first launch. The basic Shuttle Centaur vehicle, including lessons learned during its development, formed the basis of the Titan IV Centaur program with first flight in $1994^{4}$. The 16 Titan IV Centaur flights brought new CFM capabilities with the operation of three-burn missions, eight-hour missions, three-layer MLI blankets, and zero-g CFM. Titan IV Centaur still represents the state-of-the-art low boil-off for large propulsion systems ${ }^{5}$. The lessons learned from decades of Centaur development formed the basis of the Centaur III development for the Atlas III and Atlas V programs.

In an effort to increase the longevity of the existing Atlas V and Delta IV second stages, NASA Launch Services Program (LSP) initiated the EELV Evolution and Advanced Cryogenic Evolved Stage projects with the Atlas and Delta programs. These programs investigated the use of historic flight experience to guide the development of an LO2/LH2 stage that would be needed to support long mission durations (weeks, months, year). Results showed that extending storage of cryogenic propellants could be accomplished for periods up to eight months passively, and nearly unlimited time thereafter with active cooling ${ }^{7,8}$.

Improved CFM understanding will benefit the development of future EELV upper stages, EDS, Altair, propellant depots, and a wide array of additional science and national security applications. CRYOTE provides an affordable opportunity to test many critical

\begin{tabular}{|lcc|}
\hline \multicolumn{1}{|c|}{ Cryo Transfer Technology } & \multicolumn{2}{c|}{ TRL } \\
Pressure Control & $0-G$ & Settled \\
Ullage \& Liquid Stratification & 4 & 9 \\
Propellant acquisition & 3 & 9 \\
Mass Gauging & 3 & 9 \\
Propellant Expulsion Efficiency & 3 & 9 \\
System Chilldown & 3 & 8 \\
Autonomous Rendezvous \& & 7 & 7 \\
Docking & & \\
Transfer System Operation & 3 & 6 \\
Fluid Coupling & 6 & 6 \\
Passive Long Duration Storage & 5 & 5 \\
\hline $\begin{array}{l}\text { Table 1. TRLs for a selection of CFM } \\
\text { technologies }\end{array}$ \\
\hline
\end{tabular}
CFM technologies in the micro-g space environment. The benefits of CRYOTE range from stage-level demonstrations to individual CFM capabilities such as mass-gauging, liquid acquisition, and stratification management. Settled CFM operation can directly build on existing upper stage flight experience while zero-g CFM requires more advanced capabilities (Table 1 ). 


\section{B. Cryogenic System Design}

All future large cryogenic propulsion systems, including EELV upper stages, EDS, Altair, solar thermal stages and even propellant depots have several common features:

- Large cryogenic tanks (LO2 and/or LH2)

- Structural skirt attachments

- Feed and vent systems

CRYOTE's design specifically mimics these features on a modest scale. Thus CRYOTE provides a platform for testing the system design features of all of the proposed future cryogenic systems (Table 2).

\begin{tabular}{|l|l|}
\hline \multicolumn{2}{|c|}{ Cryogenic System Design } \\
\hline Technology: & CRYOTE Demonstrates: \\
\hline Low-K Adapters & $\begin{array}{l}\text { Thermal performance/expansion in a space environment } \\
\text { Localized heating accounting for boundary layer }\end{array}$ \\
\hline Low Surface Area & In-space deployment and effectiveness \\
\hline Lightweight Tank & $\begin{array}{l}\text { Impact on heating, slosh, mixing in tank: } \\
\text { - Design: smooth wall, isogrid, stringers/baffles } \\
\text { - Material: aluminum, steel, titanium, composite }\end{array}$ \\
\hline Common Bulkhead & $\begin{array}{l}\text { Acreage and wall heat transfer } \\
\text { Balance of heating for pressure control }\end{array}$ \\
\hline Internal Sump & $\begin{array}{l}\text { Allows substantial measurement to anchor models } \\
\text { Zero and low-g mass gauging }\end{array}$ \\
\hline Minimal Penetrations & $\begin{array}{l}\text { Screens, baffles, crevices, feedlines, magnets } \\
\text { Gas/liquid boundary stability in zero-g and during maneuvers }\end{array}$ \\
\hline $\begin{array}{l}\text { Table 2. CRYOTE closely mimics the generic system design of all future large scale cryogenic } \\
\text { propulsion systems on a modest scale providing the ideal in-space platform for testing system design } \\
\text { features supporting upper stages, Earth Departure Stage, Altair, and propellant depots. }\end{array}$ \\
\hline
\end{tabular}

Flight experience indicates that tank material (steel, aluminum, etc), tank mass, and tank wall geometry (smooth or iso/ortho grid) has a large impact on heat transfer, post-burn pressure spikes, and boil-off. The choice of tank material and design can have a critical impact on the ability of future cryogenic propulsion stages to meet mission requirements. CRYOTE provides an ideal platform to demonstrate the impact of these basic system-level design features on back-to-back missions.

The structural attachment of the cryogenic stage to the booster and payload can easily be the largest contributing factor to tank heating and boil-off. CRYOTE provides an opportunity to test the heat transfer of multiple attachment concepts affected by the adapter and tank conduction as well as the cryogenic fluid / wall boundary layer interaction with potential vapor formation in the micro-g space environment.

\section{CRYOTE Thermal Protection Technologies}

For long duration cryogenic fluid storage one must add specific thermal protection system (TPS) technologies to any cryogenic system (Table 3). The TPS can be as simple as MLI, similar to that flown on Titan IV Centaur, but with many more layers. The addition of using the boil-off gas to vapor-cool key heat penetrations, as well as propellant positional control to force a vapor barrier between the tank wall at high heat locations, supports long duration cryogenic storage. 


\begin{tabular}{|l|l|}
\hline \multicolumn{2}{|c|}{ Thermal Protection Technologies } \\
\hline Technology: & CRYOTE Demonstrates: \\
\hline Environment & $\begin{array}{l}\text { Vehicle orientation - Solar and Earth albedo heating } \\
\text { Atmospheric drag }\end{array}$ \\
\hline Active Cooling & $\begin{array}{l}\text { End-to-end system efficiency } \\
\text { Cooling distribution }\end{array}$ \\
\hline Sun Shield & In-space deployment and effectiveness \\
\hline Pressure Control & $\begin{array}{l}\text { Fluid stratification and mixing effectiveness } \\
\text { Effect of local heating, boundary layer impact (nucleate/film boiling and } \\
\text { heat/vapor distribution) } \\
\text { Baffles and other interior hardware } \\
\text { Zero-g, continuously settled or periodic settling, acceleration } \\
\text { TVS vs. ullage venting } \\
\text { Liquid-wall-ullage interaction (quenching/collapse) }\end{array}$ \\
\hline $\begin{array}{l}\text { Vapor-cooled Points, MLI, } \\
\text { Vapor-cool Shields }\end{array}$ & $\begin{array}{l}\text { Effectiveness following launch environment } \\
\text { Transient vent-down duration and effectiveness } \\
\text { Para-ortho conversion } \\
\text { System operation }\end{array}$ \\
\hline $\begin{array}{l}\text { Table 3. A robust thermal protection system must be added to even the best structural system design } \\
\text { to attain low tank heating and resulting boil-off. }\end{array}$ \\
\hline
\end{tabular}

MLI is a series of thin reflective sheets designed to impede radiation heat transfer, the primary mechanism in the vacuum of space. Each layer reflects heat back to its external environment. Current predictions suggest that between thirty to sixty layers of insulation may be needed to meet long duration mission requirements. The Centaur and Delta IV upper stages provide insight into the effectiveness of modest MLI shields on large cryogenic systems. However, the short duration of these stages does not allow an understanding of the steady state effectiveness of these shields once they have completely vented to vacuum. Also, the high conduction heating of these stages and limited flight data prohibit an accurate understanding of the actual tank acreage heating contributed through the shields, especially with a large number of MLI layers. Even the modest MLI shields on Titan Centaur showed mission-to-mission variation of a factor of two. Space based science cryo-dewars fly inside of a vacuum shell, with ultra low conductivity structural supports that are not practical for large cryogenic propulsion systems. Thus, the onorbit performance of thick MLI blankets designed for large cryogenic propulsion systems has not been accurately quantified. The uncertainty in installation, efficiency, and longevity requires a considerable safety factor in a fielded system. CRYOTE provides a reasonable scale opportunity to investigate the transient and steady state effectiveness of various MLI and sun shield systems under the combined solar and Earth thermal radiation environments.

Cryogenic fluid stratification directly affects the tank pressure rise associated with any given tank heat load. CRYOTE provides the opportunity to internally map stratification, providing detailed data with which to correlate thermodynamic modeling tools that will be critical to the development of future cryogenic systems. Earth-based testing does not represent the micro-g, heat transport environment on orbit, and the conservative nature of the existing payload customers prevents adding significant tank internal instrumentation to flights of existing upper stages. 


\section{CRYOTE CFM Technology Demonstration}

Long duration cryogenic storage and handling requires the combination of a well designed cryogenic system, enhanced thermal protection system, and critical CFM technologies. CRYOTE provides a very capable on-orbit laboratory with which to demonstrate the capability of these technologies (Table 4).

Effective tank system design, thermal protection and integration of TVS, liquid acquisition, and propellant mass gauging are required for Altair and the EDS missions as currently defined. MLI is required to preserve adequate cryogenic propellant for mission. A TVS is needed to control the pressure in the tank, mixing the cryogen to eliminate thermal stratification and extracting heat when mixing is no longer adequate. Liquid acquisition devices (LADs) ensure that liquid is available for reaction control propulsion systems and main engine start. Propellant mass gauging provides the final check that adequate liquid propellant is onboard prior to initiation of a propellant burn that commits the crew and spacecraft to departing Earth orbit or other subsequent mission maneuvers. CRYOTE provides a cost effective, timely solution to demonstrating these critical elements prior to committing to expensive development on the actual propulsion stage.

\begin{tabular}{|l|l|}
\hline \multicolumn{2}{|c|}{ Cryogenic Fluid Management Subsystem Technologies } \\
\hline Technology: & CRYOTE Demonstrates: \\
\hline Micro-g Mass Gauge & $\begin{array}{l}\text { Pressure-Volume-Temperature (PVT) gauge } \\
\text { Radio frequency gauge } \\
\text { Acoustic resonance gauge }\end{array}$ \\
\hline Mixing Pumps & $\begin{array}{l}\text { Destratification mixing pump - flow rate and duty cycle } \\
\text { Axial jet vs. spray bar mixing } \\
\text { Joule-Thomson valve duty cycle } \\
\text { Thermodynamic vent system duty cycle } \\
\text { Heat exchanger }\end{array}$ \\
\hline Liquid Acquisition Device (LAD) & LAD design, placement and flow capacity \\
\hline $\begin{array}{l}\text { Propellant Positional } \\
\text { Management (PPM) }\end{array}$ & $\begin{array}{l}\text { Ability to force gas or liquid boundary condition } \\
\text { Screens, baffles, crevices, feedlines, magnets } \\
\text { Gas/liquid boundary stability in zero-g and during maneuvers }\end{array}$ \\
\hline $\begin{array}{l}\text { Thermodynamic Vent System } \\
\text { (TVS) }\end{array}$ & $\begin{array}{l}\text { Ability of TVS to extract energy from cryogenic system } \\
\text { TVS effectiveness of controlling tank pressure } \\
\text { Impact of flow rates } \\
\text { Heat exchanger design }\end{array}$ \\
\hline Cryo-cooler & $\begin{array}{l}\text { Efficiency of large scale cryo-coolers } \\
\text { In-tank, tank wall, cooled MLI layer effectiveness }\end{array}$ \\
\hline Instrumentation & $\begin{array}{l}\text { Allows substantial measurement to anchor models } \\
\text { Zero-g and micro-g mass gauging }\end{array}$ \\
\hline $\begin{array}{l}\text { Table 4. On-orbit demonstration in the micro-g environment of space is critical to development of } \\
\text { promising CFM technologies. }\end{array}$ & \multicolumn{2}{|l}{} \\
\hline
\end{tabular}

Most key CFM technologies are required for any long duration flight of a cryogenic propellant system:

- Thermodynamic Vent System (TVS) - required to maintain pressure control in a cryogenic propellant tank

- Liquid Acquisition Device (LAD) - required to obtain and extract liquid from a propellant tank at micro-g conditions

- Propellant Mass Gauging - required to determine the quantity of propellant in a tank

- Fluid Transfer - required to enable multi-tank propellant systems and propellant depots 
A thermodynamic vent system (TVS) is a mechanism whereby the contents of a cryogenic tank are allowed to vent to space through a Joule-Thomson (J-T) valve and subsequent heat exchangers. Isenthalpic throttling across the J-T valve, followed by complete vaporization and further heating through the heat exchangers, provides cooling of the tank contents and interception of penetration heat leakage. A TVS may require an electric motor to mix tank contents, and the heat produced by the motor heats the propellant. Optimizing a TVS with proper duty cycle to match tank volume, surface area, internal geometry, and heat load will be difficult, if not impossible, without actual flight data.

Liquid acquisition devices (LADs) are static mechanisms that use surface tension forces to attract and hold propellant. These mechanisms are typically screens or vanes leading to a sump. The intention is to provide adequate flow to the engine feed system in a micro-g environment while not overly retarding flow during engine burn. Optimizing a LAD without flight data would be very challenging.

Propellant mass gauging is required to determine whether adequate propellant resides in a tank to perform subsequent mission requirements. This process is more straightforward under settled conditions because only the liquid level must be detected. However, under micro-g conditions, the position of the liquid is uncertain and more technologically advanced methods are required to determine propellant mass. These methods are similar in that they all attempt to measure the volume of ullage in the tank. A pressurevolume-temperature gauge uses the small displacement of a piston-like device to induce a pressure rise in the tank. Matching the pressure to the known change in volume allows for the calculation of ullage via the ideal gas law. Radio frequency and acoustic resonance both use responses of the tank's contents to measure liquid volume. Once again, micro-g conditions are required to simulate the indeterminate liquid/ullage location. Without a CRYOTE-like orbital lab, development of these micro-g mass gauging devices will be very challenging if not impossible.

Sierra Lobo's Cryo-Tracker ${ }^{\circledR}$ Mass Gauging System is in the process of being implemented on CRYOTE's first flight (Figure 11). The development of Cryo-Tracker $B$ for reliable sensing of cryogenic propellant levels and temperatures in a reduced gravity environment was undertaken by Sierra Lobo through a contract with the NASA Kennedy Space Center Launch Services Program. The Cryo-Tracker $B$ is a patented lightweight, flexible probe with multiple integral silicon diode sensing elements. It has an accuracy of $\pm 0.5 \mathrm{~K}$ and can detect liquid levels or thermal stratification by toggling between two power levels.

CRYOTE is an optimal test facility for CryoTracker®. In-flight testing of the Cryo-Tracker@ Mass Gauging System would place hardware inside of a launch vehicle propellant tank, and rocket companies are extremely reluctant to consider this as a testing option due to the increased risk to flight systems. CRYOTE provides an environment for Cryo-Tracker® to increase its Technology Readiness Level (TRL) without the risk of placing untested hardware within a flight tank.

In addition to CRYOTE being a beneficial environment for Cryo-Tracker $\AA$, Cryo-Tracker ${ }^{\circledR}$ supports CFM understanding within CRYOTE. CryoTrackerß will provide liquid level, liquid movement, and thermal stratification information within the tank.

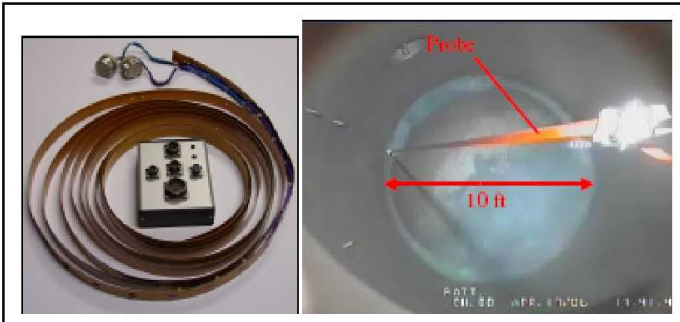

Figure 11. (Left) Cryo-Tracker@ Probe and Electronics; (Right) 33-ft Probe in Atlas LOX tank - ground test (Credit: Sierra Lobo)

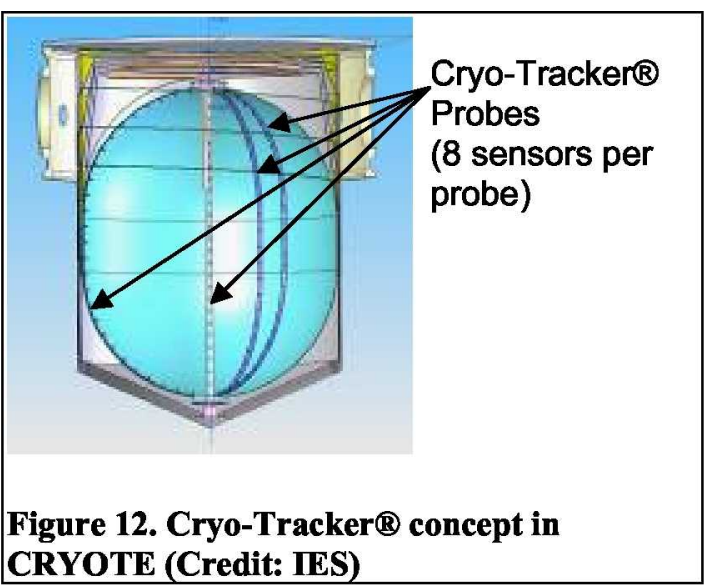

The optimal Cryo-Trackerß configuration based on the current CRYOTE concept includes four probes with eight sensors on each probe (Figure 12). Three probes are arranged at 120 degrees apart, offset from the tank wall from top to bottom. The fourth probe also reaches from the top of the tank to the bottom of the tank, but is located in the center of the tank. 
Fluid transfer between two tanks is an enabling technology for propellant depots. To be successful, it requires operating valves, chilling the receiving tank, measuring the rate of flow and the volume of propellant in the tank injecting liquid into the receiving tank, and controlling tank pressure. While groundbased demonstrations have provided significant insight into this fluid transfer process, the nuances of micro-g effects can not be adequately simulated on Earth. CRYOTE provides an end-to-end thermodynamic demonstration of the complete transfer process.

When resupplying fluids to vehicles in orbit, the transfer of liquid between tanks is not straightforward because of the micro-g environment. To maintain a predictable environment during transfer, only gas (with no liquid) can pass through a vent, so the normal procedure of venting a tank as it is filled is not applicable because of the location uncertainty of liquid vs. gas. An alternative is to perform a no-vent transfer, and this operation is currently in the trade space for the transfer of LH2 to the CRYOTE tank.

Two approaches to transferring cryogenic fluid from one tank to another without venting were investigated in 1986 by Martin Marietta ${ }^{9}$. One uses the kinetic transfer energy to promote random mixing within the receiver tank to achieve required heat transfer. The other relies on centrifugal positioning and a fine spray of liquid to achieve the same purpose. The latter approach appears to offer the advantage of being less dependent on micro-g mechanisms, and is not restricted by tank size.

Fluid and thermal analysis showed that material properties greatly affect no-vent fill, with $\mathrm{LH} 2$ and liquid helium being the fluids most likely to have transfer rate issues. The assessments conducted required a significant number of assumptions concerning the in-tank processes influenced by a micro-g environment. Test data is not currently available to quantify the processes, or even to verify the mechanisms that are important. Acquiring the data needed requires a micro-g test environment of sufficient duration to indicate steady-state behavior, such as CRYOTE.

\section{E. Propellant Depots}

For demanding missions beyond LEO, propellant makes up the vast majority of the required LEO launch mass. Mission designers can maximize the exploration mission architectures by choosing to load the propulsion systems on orbit from orbital stations called propellant depots. Propellant depots provide a huge force multiplier, benefiting all Exploration architectures. For example, propellant in NASA's lunar mission architecture makes up $75 \%$ of the initial mass in LEO.

Cryogenic propellant depots can be developed in a variety of configurations, from relatively simple configurations ${ }^{10}$ (Figure 13) to more complicated space stations (Figure 14). To avoid high up-front risk/costs and lengthy development periods, the development and implementation of propellant depots follow a natural technology/market driven progression:

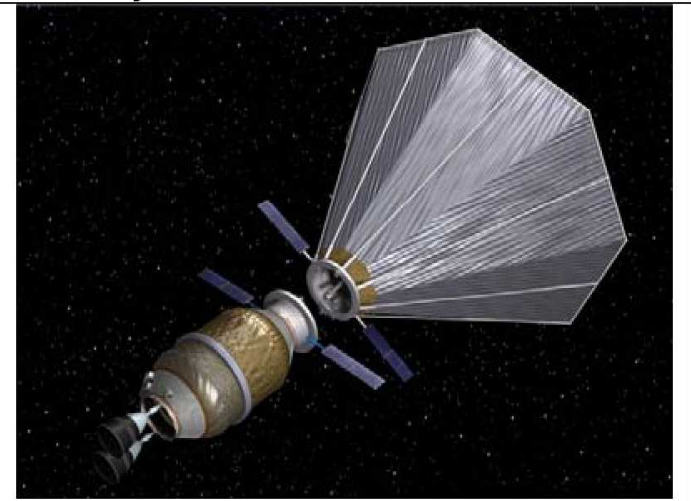

Figure 13. Single launch simple propellant depot concept that can use settled CFM to enable near term operation.

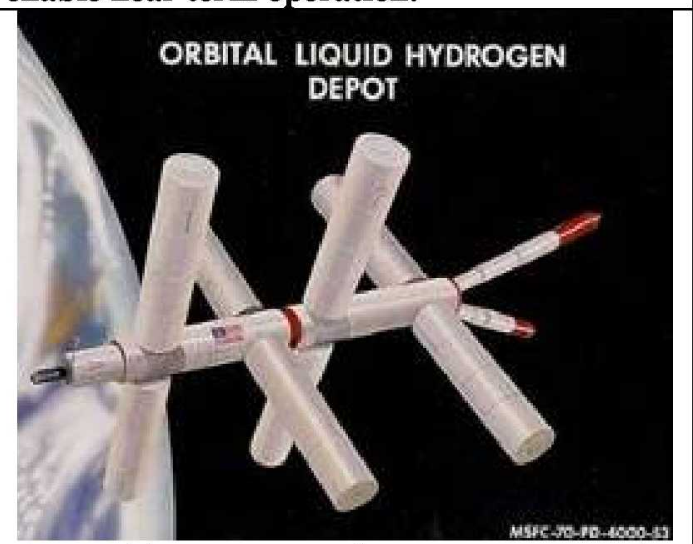

Figure 14. Large, multi-launch space station style depots typically rely on zero-g CFM. (Credit: NASA)

1. Near term flight demonstration

2. Large scale, depot hardware in the loop demo

3. Simple propellant depots

4. Robust depot infrastructure

CRYOTE provides the near term, end-to-end demonstration of the propellant depot concept, including chilldown, transfer, and long-term storage of cryogenic fluids. CRYOTE can also increase the TRL of critical CFM systems such as TVS, mixing pump, LAD, propellant mass gauging, and cryogenic fluid 
coupling. CRYOTE can increase component and system TRL to Level 7. It may be desirable to follow CRYOTE with a large scale depot prototype demonstration incorporating all of the CFM systems prior to launching an actual depot.

\section{F. NASA/Industry Collaboration}

A benefit of the CRYOTE project that is not directly related to test results or technology advancement is the alliance of contributors across industry and NASA, working toward the common goal of advancing the understanding of CFM technologies. ULA has managed the project and developed launch vehicle interface details and mission integration requirements. Innovative Engineering Solutions (IES) has led the technical design of the CRYOTE spacecraft and prototype development, and is preparing the test setup for the ground prototype demonstration. NASA Kennedy Space Center, Glenn Research Center, and Marshall Space Flight Center have contributed to system definition and have conducted thermal and fluid analysis in support of the CRYOTE system. The NASA Cryogenics Group consisting of members from across NASA are identifying experiments that can utilize the CRYOTE system. NASA Joint Propulsion Lab donated flight-weight tank hardware. Lockheed Martin has provided design and operation support using their extensive experience designing long-duration cryogenic dewars. Sierra Lobo has been an active partner by providing their Cryo-Tracker® micro-g mass gauging system and associated integration expertise.

In recent years, competition between companies and differing solutions to the development of space exploration has made collaboration difficult between entities across industry and government. The CRYOTE project has bridged many relationships with the common goal of advancing technologies that can benefit current short-term cryogenic missions as well as support a robust architecture for future long-term, high-performance missions.

\section{Rideshare Opportunities}

EELV missions with at least $1000 \mathrm{lbm}$ of performance excess can accommodate the weight of CRYOTE, with enough excess LH2 to fill CRYOTE's tank after the primary payload is separated. ULA is tracking propellant margins for future missions. The precision of ULA launches potentially allows CRYOTE to launch on missions with a published performance margin of less than $1000 \mathrm{lbm}$. If actual launch performance is off-nominal, which is rare, then CRYOTE will simply be filled to less than full capacity.

The majority of EELV missions have one of two customers - the United States Department of Defense or NASA. ULA is working with the DoD's Space Test Program (STP) and NASA Launch Services Program (LSP) to explore Rideshare options.

NASA will gain insight into critical CFM technologies required to support NASA's Exploration Program with CRYOTE. Therefore, it stands to reason that a NASA mission would be the most appropriate mission on which to fly CRYOTE. Landsat, the only NASA mission in the next few years that will stay in Earth's orbit and has enough performance excess for a demonstration of the system chilldown, fluid transfer, and technology mass gauging of CRYOTE, is scheduled for launch in late 2012. This demonstration would provide a proof of concept that CRYOTE's reverse settling sequence can successfully transfer LH2 from Centaur to the experiment tank. The proof of concept mitigates risk to give CRYOTE a better chance for permission to fly on DoD missions, of which there are several potentially compatible missions forecasted in the next few years. If CRYOTE could fly as an auxiliary payload with the Global Positioning System (GPS) or Defense Meteorological Satellite Program (DMSP) satellites, these recurring missions would allow for follow-on CRYOTE flights without repeating the required detailed mission integration analysis that is required for new missions. Recurring flights provide the ability to conduct incrementally more complex CFM experiments using the testbed. 


\section{Conclusion}

Cryogenic propellants have proven over many decades to be a powerful and reliable tool for accomplishing high performance missions. The enhancement of CFM technologies can enable longduration use and increase handling capability of these high-performance propellants in near-term designs that will shape future space transportation architecture. The efficiency of launch vehicle upper stages impacts the mass constraints of national security satellites. Achieving high performance in long-duration vehicles such as EDS and Altair impacts the number of people and infrastructure that can be transported to the moon and beyond. A better understanding of CFM in spacecraft dewars can enhance Earth observation and our understanding of the cosmos. Propellant depots, for which CRYOTE demonstrates end-to-end system CFM management including system chilldown and transfer, handling, and storage of cryogenic propellants, can provide a foundation for a robust space transportation architecture.

Because the space industry is largely risk averse to incorporating unproven systems or conducting experiments using flight hardware supporting a primary mission, CRYOTE provides an unmatched opportunity to advance in-space CFM understanding at an affordable cost. At a minimum, CRYOTE demonstrates system chilldown, cryogenic propellant transfer and handling, pressure control, boil-off rate, thermal stratification, and mass gauging at micro-g during its first flight. The testbed can also accommodate experiments demonstrating mixing, active cooling, and propulsive concepts. The high performance margin of EELV launch vehicles and CRYOTE's straightforward testbed design provides an affordable platform to increase CFM knowledge, reduce technical risks, and ultimately support the design of long-duration cryogenic vehicles that could close the gap between Earth and the rest of our solar system. 


\section{References}

${ }^{1}$ Bernard F. Kutter, Frank Zegler, Jon Barr, Tim Bulk, Brian Pitchford, "Robust Lunar Exploration Using an Efficient Lunar Lander Derived from Existing Upper Stages", AIAA 2009-6566.

${ }^{2}$ Chato, D., "The role of flight experiments in the development of cryogenic fluid management technologies," Cryogenics, Vol. 46, Issues 2-3, 2005 Space Cryogenics Workshop, Feb-March 2006, Pages 82-88, ISSN 0011-2275 URL: http://www.sciencedirect.com/science/article/B6TWR-4J021X31/2/785e6478b5a05921b92fc6d255159db7 [cited 31 July 2009].

3 "Titan/Centaur Flight Evaluation TC-5", February 1977, Contract NAS3-19154, Report \# CASD/LVP76-066.

4 "History of the Titan Centaur Launch Vehicle", http://www.ulalaunch.com/docs/publications/HistoryoftheTitanCentaurLaunchVehicle.pdf [cited 17 August 2009].

${ }^{5}$ Jeffrey S. De Kruif, "Centaur Upper Stage Applicability for Several-Day Mission Durations with Minor Insulation Modifications", AIAA 2007-5845, http://www.ulalaunch.com/docs/publications/CentaurUpperstageApplicabilityforSeveralDayMissionD urationswithMinorInsulationModificationsAIAA20075845.pdf [cited 17 August 2009], September 2007.

${ }^{6}$ Thomas J. Rudman, "The Centaur Upper Stage Vehicle”, http://www.ulalaunch.com/docs/publications/TheCentaurUpperStageVehicleHistory.pdf [cited 17 August 2009], December 2002.

${ }^{7}$ Bernard F. Kutter, "Atlas Centaur Extensibility to Long-Duration In-Space Applications", http://www.ulalaunch.com/docs/publications/AtlasCentaurExtensibilitytoLongDurationInSpaceApplic ations20056738.pdf [cited 17 August 2009], AIAA 2005-6738,September 2005.

${ }^{8}$ Gerard Szatkowski, "Centaur Extensibility for Long Duration", http://www.ulalaunch.com/docs/publications/CentaurExtensibilityForLongDuration20067270.pdf [cited 17 August 2009], AIAA-2006-7270, September 2006.

${ }^{9}$ Gill, J.P., "Analysis and Modeling of Fluid Transfer in Orbit," AIAA-86-1718, 1986.

${ }^{10}$ Bernard F. Kutter, "A Practical, Affordable Cryogenic Propellant depot Based on ULA's Flight Experience", AIAA 2008-7644, http://www.ulalaunch.com/docs/publications/APracticalAffordableCryogenicPropellantDepotBasedon ULAsFlightExperience20087644.pdf [cited 17 August 2009], September 2008 